\title{
Stability for a Class of Equilibrium Solutions to the Coagulation-Fragmentation Equation
}

\author{
Wilson Lamb and Iain W Stewart \\ Department of Mathematics, University of Strathclyde, Glasgow, G1 1XH, UK
}

\begin{abstract}
We consider the behaviour of solutions to the continuous constant-rate coagulation-fragmentation equation in the vicinity of an equilibrium solution. Semigroup methods are used to show that the governing linear equation for a perturbation $\varepsilon(x, t)$ has a unique globally defined solution for suitable initial conditions. In addition, Laplace transforms and the method of characteristics lead to an explicit formula for $\varepsilon$. The long-term behavior of $\varepsilon$ is also discussed.
\end{abstract}

Keywords: coagulation-fragmentation; stability of equilibria

PACS: $02.60 . \mathrm{Nm}, 36.40 . \mathrm{Qv}$

\section{INTRODUCTION}

We examine the continuous coagulation-fragmentation equation (CFE)

$$
\frac{\partial u}{\partial t}(x, t)=\frac{F}{2}\left(-x u(x, t)+2 \int_{x}^{\infty} u(y, t) d y\right)+\frac{K}{2}\left(\int_{0}^{x} u(x-y, t) u(y, t) d y-2 u(x, t) \int_{0}^{\infty} u(y, t) d y\right),
$$

with initial data given by

$$
u(x, 0)=u_{0}(x) \geq 0 .
$$

Equation (1) describes the time evolution of the concentration of particles $u(x, t) \geq 0$ of size $x \geq 0$ at time $t \geq 0$ whose change in mass is governed by the constant reaction rates $K>0$ and $F>0$ which are called, respectively, the coagulation and fragmentation kernels.

Investigations into (1) and (2) have a long history. For example, in 1979 Aizenman and Bak [1] used semigroup methods to establish global existence and uniqueness of nonnegative, mass-conserving solutions satisfying

$$
\int_{0}^{\infty}(1+x) u(x, t) d x<\infty, \text { for all } t>0 .
$$

An alternative approach, involving compactness arguments, has also been used by Stewart [2] to prove the global existence of solutions, satisfying (3), for CFEs in which the kernels are not necessarily constant. Note that condition (3) guarantees that, for all $t \geq 0$, the total number of particles in the system, $N(t):=\int_{0}^{\infty} u(x, t) d x$, and the total mass in the system, $M(t):=\int_{0}^{\infty} x u(x, t) d x$, are both finite.

As the long-term behaviour of such solutions is also of interest, there have been a number of studies aimed at determining what happens to $u(x, t)$ as $t \rightarrow \infty$. The asymptotic behaviour of solutions to (1) and (2) was considered initially in [1], and then investigated further, via the invariance principle, in a later paper [3] by Stewart and Dubovskii. It was shown that, as $t \rightarrow \infty$, each time-dependent solution $u(x, t)$ approaches an equilibrium

$$
u_{M}(x)=\lambda \exp (-x \sqrt{\lambda / M}), \quad \lambda=F / K,
$$

that is uniquely determined by the initial mass $M=\int_{0}^{\infty} x u_{0}(x) d x\left(=\int_{0}^{\infty} x u(x, t) d x\right)$.

In [1], where $F=K=2$, the behaviour of solutions in the vicinity of a typical equilibrium was examined by setting

$$
u(x, t)=u_{M}(x)+\psi(x, t) \exp \left(-M^{-1 / 2} x / 2\right),
$$

and then obtaining an approximate linear equation for $\psi$ which was studied in the Hilbert space $L_{2}([0, \infty))$. In the present paper we re-examine the behaviour of solutions in the vicinity of the equilibrium $u_{M}$, but work instead within the more physical setting of the Banach space

$$
X:=\left\{\phi:\|\phi\|_{X}:=\int_{0}^{\infty}(1+x)|\phi(x)| d x<\infty\right\},
$$


and use a perturbation of the form

$$
u(x, t)=u_{M}(x)+\varepsilon(x, t) .
$$

The resulting (approximating) linear equation for $\varepsilon$,

$$
\frac{\partial \varepsilon}{\partial t}(x, t)=\frac{F}{2}\left(-x \varepsilon(x, t)+2 \int_{x}^{\infty} \varepsilon(y, t) d y\right)+K\left(\int_{0}^{x} u_{M}(x-y) \varepsilon(y, t) d y-u_{M}(x) \int_{0}^{\infty} \varepsilon(y, t) d y-\varepsilon(x, t) \int_{0}^{\infty} u_{M}(y) d y\right),
$$

is shown to have a unique global solution such that $\varepsilon(x, t) \in X$ for each $t$. An explicit formula for $\varepsilon$ is also obtained by using Laplace transform techniques and the method of characteristics.

\section{THE GOVERNING PERTURBATION EQUATION}

To obtain the governing perturbation equation (6), we assume that, in the vicinity of of an equilibrium $u_{M}$, the solution of (1) and (2) takes the form

$$
u(x, t)=u_{M}(x)+\varepsilon(x, t),
$$

where $\varepsilon$ is a small perturbation to the equilibrium solution $u_{M}$ and $\int_{0}^{\infty} x u_{0}(x) d x=M$. Substituting into (1), and neglecting terms involving products of $\varepsilon$, leads to the linear equation (6). Consequently, if $\varepsilon$ is small and satisfies (6) then $v(x, t):=u_{M}(x)+\varepsilon(x, t)$ should be a good approximation to the solution $u$.

To enable semigroup methods to be applied to (6), we note first that the initial-value problem (1) and (2) can be expressed in abstract form as

$$
\frac{d}{d t} u(t)=G[u(t)]+N[u(t)], t>0 ; \quad u(0)=u_{0},
$$

where the nonlinear coagulation operator $N$ is given by

$$
(N \phi)(x):=\frac{K}{2} \int_{0}^{x} \phi(x-y) \phi(y) d y-K \phi(x) \int_{0}^{\infty} \phi(y) d y, \quad \phi \in X,
$$

and $G$ is an operator realisation of the mapping

$$
\phi(x) \rightarrow-\frac{F}{2} x \phi(x)+F \int_{x}^{\infty} \phi(y) d y
$$

that generates a strongly continuous semigroup of operators, $\{\exp (t G)\}_{t \geq 0}$, on $X$; see [4] for details. As the Fréchet derivative of $N$ at $f \in X$ is given by

$$
\left(N_{f} \phi\right)(x)=K\left(\int_{0}^{x} f(x-y) \phi(y) d y-f(x) \int_{0}^{\infty} \phi(y) d y-\phi(x) \int_{0}^{\infty} f(y) d y\right),
$$

for all $\phi \in X$, it follows that the abstract version of the initial-value problem associated with (6) takes the form

$$
\frac{d}{d t} \varepsilon(t)=G[\varepsilon(t)]+N_{u_{M}}[\varepsilon(t)], t>0 \varepsilon(0)=\varepsilon_{0} .
$$

From [5, Theorem 2.32] and the fact that $N_{u_{M}}$ is a bounded linear operator on $X$, we deduce that $L=G+N_{u_{M}}$ is the infinitesimal generator of a strongly continuous semigroup $\{\exp (t L)\}_{t>0}$ on $X$ and so, for suitable $\varepsilon_{0}$, (11) has a unique strongly-differentiable solution $\varepsilon:[0, \infty) \rightarrow X$ given by $\varepsilon(t)=\exp (t L) \varepsilon_{0}$. Moreover, on setting $\varepsilon(x, t)=[\varepsilon(t)](x)$, it can be shown that, when $\varepsilon_{0}$ satisfies the mass-conserving condition $\int_{0}^{\infty} x \varepsilon_{0}(x) d x=0$, then, for each $t>0$,

$$
\int_{0}^{\infty} x \varepsilon(x, t) d x=0
$$

and

$$
\int_{0}^{\infty} \varepsilon(x, t) d x=\left(\int_{0}^{\infty} \varepsilon_{0}(x) d x\right) \exp \left(-K t \int_{0}^{\infty} u_{M}(x) d x\right)
$$




\section{EXPLICIT SOLUTIONS TO THE PERTURBATION EQUATION}

\section{Case (i) $K(N(0))^{2}=F M$}

One interesting feature observed in [3] is that $N(t)=N(0)$, for all $t>0$, whenever the initial data $u_{0}$ satisfies the constraint $K(N(0))^{2}=F M$. In this case, it is natural to require that the initial perturbation satisfies

$$
\int_{0}^{\infty} \varepsilon_{0}(x) d x=\int_{0}^{\infty} x \varepsilon_{0}(x) d x=0 .
$$

It then follows from (4) and (13) that the governing linear perturbation equation (6) reduces to

$$
\frac{\partial \varepsilon}{\partial t}(x, t)=-2 b \varepsilon(x, t)\left[\frac{1}{a}+\frac{x}{2}\right]+2 b \int_{0}^{x}\left(e^{-a(x-y)}-1\right) \varepsilon(y, t) d y,
$$

subject to the initial condition

$$
\varepsilon(x, 0)=\varepsilon_{0}(x),
$$

where

$$
a=\sqrt{\frac{F}{K M}}, \quad b=\frac{F}{2} .
$$

On applying the Laplace transform to equation (15) we obtain

$$
\frac{\partial \hat{\varepsilon}}{\partial s}(s, t)-\frac{1}{b} \frac{\partial \hat{\varepsilon}}{\partial t}(s, t)=2\left(\frac{1}{a}+\frac{1}{s}-\frac{1}{s+a}\right) \hat{\varepsilon}(s, t),
$$

where $\hat{\varepsilon}(s, t)$ denotes the Laplace transform of $\varepsilon(x, t)$. The method of characteristics then leads to

$$
\begin{aligned}
\hat{\varepsilon}(s, t)=\hat{\varepsilon}_{0}(s+b t) e^{-2 b t / a}\left[1+\frac{(a b t)^{2}}{(b t-a)^{2}}\right. & \left(\frac{1}{(s+b t)^{2}}+\frac{1}{(s+a)^{2}}\right) \\
& +2 a b t \\
& \left.\frac{\left(a^{2}-a b t+b^{2} t^{2}\right)}{(b t-a)^{3}}\left(\frac{1}{s+b t}-\frac{1}{s+a}\right)\right] .
\end{aligned}
$$

The inverse Laplace transform of (19) can now be obtained using standard formulae for convolutions and shifts, and results in

$$
\begin{aligned}
\varepsilon(x, t)=e^{-2 b t / a}[ & e^{-b t x} \varepsilon_{0}(x)+\frac{(a b t)^{2}}{(b t-a)^{2}} \int_{0}^{x}(x-y)\left(e^{-b t(x-y)}+e^{-a(x-y)}\right) e^{-b t y} \varepsilon_{0}(y) d y \\
& \left.+2 a b t \frac{\left(a^{2}-a b t+b^{2} t^{2}\right)}{(b t-a)^{3}} \int_{0}^{x}\left(e^{-b t(x-y)}-e^{-a(x-y)}\right) e^{-b t y} \varepsilon_{0}(y) d y\right] .
\end{aligned}
$$

It can be verified that $\varepsilon(x, t)$ is finite and continuous at $t=a / b$, so that the solution in (20) is continuous for all $x \geq 0$ and $t \geq 0$ if $\varepsilon_{0}$ is continuous. It is also seen from (20) that, pointwise in $x$,

$$
\varepsilon(x, t) \rightarrow 0 \quad \text { as } \quad t \rightarrow \infty .
$$

Hence the equilibrium solution is linearly stable provided (14) holds.

$$
\text { Case (ii) } K(N(0))^{2} \neq F M
$$

In this case, $N(t)$ is no longer constant, but instead is given by the formula

$$
N(t)=\frac{2 a M(N(0)+a M)}{N(0)+a M+(a M-N(0)) e^{-2 b t / a}}-a M .
$$


The Laplace transform of equation (6) gives

$$
\frac{\partial \varepsilon}{\partial s}(s, t)-\frac{1}{b} \frac{\partial \varepsilon}{\partial t}(s, t)=2\left(\frac{1}{a}+\frac{1}{s}-\frac{1}{s+a}\right) \varepsilon(s, t)+2\left(\frac{1}{s+a}-\frac{1}{s}\right) g(t)
$$

where

$$
g(t)=\int_{0}^{\infty} \varepsilon(y, t) d y .
$$

This equation is similar to that in (18) except for the additional terms on the right-hand side, and as before, we can obtain a solution by the method of characteristics. In this case, we arrive at

$$
\begin{aligned}
\hat{\varepsilon}(s, t)=\hat{\varepsilon}_{0}(s+b t) e^{-2 b t / a} & \frac{(s+a+b t)^{2}}{(s+b t)^{2}} \frac{s^{2}}{(s+a)^{2}} \\
+ & 2 a \frac{s^{2}}{(s+a)^{2}} \int_{0}^{b t} g\left(\frac{b t-y}{b}\right) e^{-2 y / a} \frac{(y+s+a)}{(y+s)^{3}} d y .
\end{aligned}
$$

From (13), $g(t)=B \exp (-2 b t / a)$, where $B=\int_{0}^{\infty} \varepsilon_{0}(x) d x$, and therefore equation (25) becomes

$$
\begin{aligned}
\hat{\varepsilon}(s, t)=\hat{\varepsilon}_{0}(s+b t) e^{-2 b t / a} \frac{(s+a+b t)^{2}}{(s+b t)^{2}} \frac{s^{2}}{(s+a)^{2}} \\
+2 a B e^{-2 b t / a} \frac{s^{2}}{(s+a)^{2}}\left[\frac{a}{2}\left(\frac{1}{s^{2}}-\frac{1}{(s+b t)^{2}}\right)+\frac{1}{s}-\frac{1}{s+b t}\right] .
\end{aligned}
$$

Consequently,

$$
\begin{aligned}
\varepsilon(x, t)=e^{-2 b t / a}\left[e^{-b t x} \varepsilon_{0}(x)+\frac{(a b t)^{2}}{(b t-a)^{2}} \int_{0}^{x}(x-y)\left(e^{-b t(x-y)}+e^{-a(x-y)}\right) e^{-b t y} \varepsilon_{0}(y) d y\right. \\
+2 a b t \frac{\left(a^{2}-a b t+b^{2} t^{2}\right)}{(b t-a)^{3}} \int_{0}^{x}\left(e^{-b t(x-y)}-e^{-a(x-y)}\right) e^{-b t y} \varepsilon_{0}(y) d y \\
+a B \frac{b t}{(a-b t)^{3}}\left\{e^{-a x}\left(2 b t(a-b t)-2 a^{2}-a b t x(a-b t)\right)\right. \\
\left.\left.+e^{-b t x}\left(2 b t(b t-a)+2 a^{2}-a b t x(a-b t)\right)\right\}\right] .
\end{aligned}
$$

Once again it can be shown that $\varepsilon(x, t)$ has a finite limit as $t \rightarrow a / b$, and $\varepsilon(x, t) \rightarrow 0$ (pointwise in $x$ ) as $t \rightarrow \infty$.

\section{REFERENCES}

1. M. Aizenman and T. A. Bak, Comm. Math. Phys, 65, 203-230 (1979).

2. I. W. Stewart, Math. Meth. Appl. Sci., 11, 627-648 (1989).

3. I. W. Stewart and P. B. Dubovskii, Math. Meth. Appl. Sci., 19, 171-185 (1996).

4. W. Lamb, Math. Meth. Appl. Sci., 27, 703-721 (2004).

5. A. Belleni-Morante and A. C. McBride, Applied Nonlinear Semigroups, Wiley, Chichester, 1998. 